\title{
Título de la síntesis: Aprendizaje por indagación: su importancia en el desarrollo de la competencia científica
}

\author{
Título completo de la tesis de posgrado: Análisis del impacto del \\ modelo didáctico de aprendizaje por indagación en biología, sobre el \\ desarrollo de la competencia científica en estudiantes de educación \\ secundaria
}

Palabras clave: Proyectos, indagación, competencia científica, investigación-acción.

Daisy Imbert Romero daisyimbertromero@gmail.com ORCID 0000-0002-2821-2844

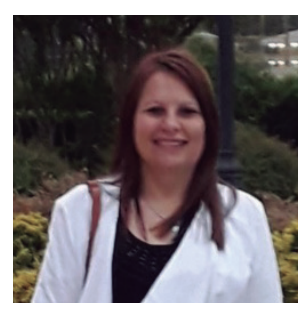

Director de tesis: Eduardo Elósegui Bandera

Institución donde se realizó el trabajo de tesis: Liceo Ciclo Básico. Paso de los Toros. Uruguay.

Fecha defensa de la tesis: 23 de setiembre de 2020

Acceso a texto completo: http://repositorio.cfe.edu. uy/handle/123456789/1337

Nombre de la Carrera de posgrado: Doctorado en Educación: Línea de investigación en aprendizaje y educación. Factores y estrategias asociados.

Institución y/o programa donde emitió el título: Universidad Internacional Iberoamericana.

\section{Síntesis del trabajo}

Frente al problema de falta de interés de los estudiantes en el aprendizaje de las ciencias, se planteó una investigación mixta, longitudinal, de investigación-acción, enmarcada en un enfoque socio-crítico, que buscó conocer la realidad y transformarla. Se complementó con un enfoque cuantitativo al trabajar con grupos control y una encuesta.
El objetivo general fue analizar el impacto del aprendizaje por indagación en el desarrollo de la competencia científica cuando se abordan los contenidos de Biología en los cursos de primero, tercero y cuarto año de enseñanza media.

La competencia científica quedó definida tomando las dimensiones mencionadas en el marco de las Pruebas PISA y las indicadas por Franco Mariscal (2015): 1. identificación de cuestiones científicas, explicar fenómenos científicamente y utilizar evidencia científica, 2) identificación de problemas científicos, definir los objetivos y formular hipótesis, 3) buscar información de diferentes fuentes y valorarla de forma crítica y reflexiva, 4. identificar variables, diseñar una metodología, realizar experiencias, 5. observar sistemáticamente, seleccionar y emplear el instrumento de medida más adecuado, procesar los resultados en distintos formatos (tablas, gráficos). 6. interpretar los resultados, formular conclusiones, 7. dar a conocer los resultados, 8. interesarse por problemas científicos, valoración del enfoque científico y conciencia ambiental.

Reflexionar de forma crítica sobre los resultados, trabajar en equipo, respetar y valorar las ideas de los compañeros y tomar decisiones.9. identificar prácticas científicas beneficiosas para la mayoría de los ciudadanos, participar como integrantes de una comunidad en la reflexión colectiva a partir del proceso de investigación.

La investigación se realizó en un liceo de contexto sociocultural desfavorable, en la ciudad de Paso de los Toros, Uruguay. Participaron tres docentes, seleccionadas por muestreo casual por estar dispuestas a participar de la investigación- acción, quienes escogieron el mejor grupo y el de mayor dificultad al inicio del año lectivo, de acuerdo al diagnóstico realizado. Intervinie- 
ron 115 estudiantes, pertenecientes a dos primeros, dos terceros y dos cuartos y 36 estudiantes participaron en los grupos testigos, correspondientes a un primer y tercer año.

Las técnicas aplicadas fueron: la observación de 18 clases, 12 grupos focales de docentes y estudiantes y una encuesta final a docentes y estudiantes, el análisis de documentos comprendidos por 243 rúbricas y 30 pósteres. Las rúbricas formaron parte de los instrumentos correspondientes a los pre test y post test, aplicados a los grupos experimentales y de control. Los instrumentos fueron validados por expertos y se realizó un pre testeo.

Se llevó a cabo una investigación-acción en tres ciclos. Se partió de un diagnóstico correspondiente al pre test, se realizó la intervención en la que se planificaron las secuencias didácticas con las docentes en base a proyectos de indagación por unidad curricular, se observó la implementación de lo planificado asistiendo a las clases y se reflexionó junto a estudiantes y docentes utilizando los grupos focales lo que constituyó el primer ciclo". De este trabajo, surgieron aspectos que se deberían mejorar para la próxima secuencia que dio lugar al segundo ciclo. Este procedimiento se reiteró en tres ciclos, al finalizar esta etapa se organizó una jornada para presentar los proyectos, para lo cual se elaboraron posters. Luego la investigadora se retiró del trabajo de campo. Posteriormente, las docentes continuaron con otras secuencias basadas en proyectos. Al finalizar el año lectivo se aplicó el post test y la encuesta.

Los datos obtenidos de las evaluaciones se analizaron utilizando el software SPSS. Para el análisis de los grupos focales se utilizó el programa "Atlas ti". Se "triangularon" los análisis cualitativos y cuantitativos, sin olvidar que, una mayor validez en la investigación cualitativa, surge de la transformación que logra.

Se presenta una síntesis de los resultados obtenidos respecto a uno de los objetivos específicos, el correspondiente al desarrollo de la competencia científica lograda por los estudiantes, cuya evidencia surge de la aplicación del pre test y post test.

En la evaluación final, los seis grupos, coinciden en los resultados aceptables en tres de las dimensiones de la competencia científica: 1) identificación de cuestiones científicas, explicar fenómenos científicamente y utilizar evidencia científica, 2) identificación de problemas científicos, definir los objetivos y formular hipótesis, 3) trabajar en equipo y tomar decisiones.
Las dimensiones con peores resultados fueron: procesar los resultados en distintos formatos y formular conclusiones que sólo lograron los estudiantes de cuarto año. En tanto otras dimensiones como buscar información de diferentes fuentes y valorarla de forma crítica y reflexiva; identificar variables, diseñar una metodología, realizar experiencias; dar a conocer los resultados e Identificar prácticas científicas beneficiosas para la mayoría de los ciudadanos son logradas sólo por estudiantes de tercero y cuarto año liceal.

Cuando se analiza si existe asociación entre la variable curso y la variable dimensión, tomando en cuenta su distribución en una tabla de contingencia (Crosstab) mediante el $\chi^{2}$, a través de la pregunta en todos los análisis de relación entre el curso y la calificación obtenida por los alumnos, los resultados indican que, en las nueve dimensiones de la competencia científica evaluada, se rechaza la $\mathrm{H} 0$, por lo que no se distribuyen al azar. Lo mismo ocurre cuando se analiza la relación entre el grupo (experimental/control) y la calificación obtenida por los estudiantes en este indicador. En los nueve indicadores, se identifica que la distribución entre insuficientes y aprobados, se corresponde con un porcentaje de insuficientes mayor en el grupo control frente al grupo experimental.

Además, cuando se comprueba si existen diferencias entre los distintos cursos y las puntuaciones obtenidas por los participantes en el global de las pruebas y en cada uno de los indicadores, mediante las pruebas: $F$ (Anova) para el contraste paramétrico y U de MannWhitney para el contraste no paramétrico, usando el test $d$ homogeneidad de varianzas mediante la prueba de Levene, el análisis de las diferencias entre los grupos de primer año, tercero y cuarto, indica que mejoran significativamente todos los cursos en la puntuación total de la prueba al compararse los resultados obtenidos antes de la intervención y después de la misma, como puede apreciarse en la figura 1.

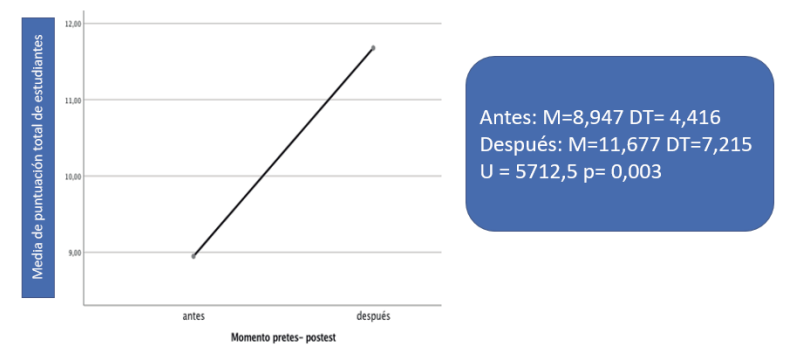

Figura 1. Diferencias significativas en la puntuación obtenida por los participantes antes y después de la intervención, con independencia del grupo al que pertenecen. 
En referencia al avance que logran realizar los estudiantes pudieron constatarse diferencias en los tres niveles.

En primer año mejoran significativamente ambos cursos en la puntuación total de la prueba: 10-1 pasa de obtener una puntuación media de 7,11 con $\mathrm{Dt}=4,35$, a una puntuación media de 10,76 con $D t=6,14$. El curso 10-3 pasa de obtener una puntuación media de 5,95 con $\mathrm{Dt}=4,73$, a una puntuación media de 11,64 con $\mathrm{Dt}=4,48$. Esta mejora se produce igualmente en ambos cursos para las dimensiones 1 y 2 .

En el curso 1 - 1, no se producen más mejoras; en cambio, en el curso 10-3 se producen avances estadísticamente significativos en dos las dimensiones

En tercer año mejoran significativamente ambos cursos en la puntuación total de la prueba: 3-2 pasa de obtener una puntuación media de 10,941 con Dt= 3,960 a una puntuación media de 14,571 con Dt= 4,362 .El curso 3--3 pasa de obtener una puntuación media de 9,312 con $\mathrm{Dt}=2,358$, a una puntuación media de 11,786 con $\mathrm{Dt}=3,945$

Esta mejora se produce igualmente en ambos cursos para la dimensión 2. En el curso 3-2, se producen mejoras estadísticamente significativas en la dimensión 9, en cambio, en el curso 3--3 se producen mejoras estadísticamente significativas en las dimensiones 3 y 4.

En tanto para 4to año mejoran significativamente ambos cursos en la puntuación total de la prueba: 4ㅇ--1 pasa de obtener una puntuación media de 11,260 con $\mathrm{Dt}=4,464$ a una puntuación media de 19,687 con Dt= 4,174 . El curso 4--2 pasa de obtener una puntuación media de 8,952 con $\mathrm{Dt}=3,681$, a una puntuación media de 20,692 con $\mathrm{Dt}=4,571$.

Esta mejora se produce igualmente en ambos cursos para la dimensión 1, 2, 3, 5, 6 y 7. En el curso 4ㅇ--1, se producen además mejoras estadísticamente significativas en la dimensión 4; en cambio, en el curso 4--2 se producen mejoras estadísticamente significativas en la dimensión 9, además de las mencionadas anteriormente para ambos grupos.
Los grupos de control a los que se aplicó la misma prueba final obtuvieron resultados muy bajos.

Fue posible planificar el curso en secuencias basadas en proyectos de indagación. Se logró implementar las mismas atendiendo a los contenidos conceptuales del currículum, promoviendo la problematización a partir del contexto, así como contemplando los intereses de los estudiantes.

Por otra parte, el análisis de $\chi^{2}$ para los diferentes grupos y en la comparación con grupos control, así como el estudio de diferencias entre medias, antes y después de la intervención, indican resultados estadísticamente significativos, que fueron distintos de acuerdo al grado escolar. Se obtienen mejores resultados en cuarto año, mientras que tercer año logra promedios superiores respecto a primero, de acuerdo a las dimensiones de la competencia científica en las que obtienen mejor puntuación, en cada nivel, al comparar el pre test y post test.

Puede apreciarse que algunas dimensiones se desarrollan mejor que otras luego de trabajar todo el año lectivo a través de proyectos de indagación. Si bien todos los grupos mejoran de forma estadísticamente significativa, los grupos de cuarto año logran dicha mejora en mayor cantidad de dimensiones respecto a primero y tercero.

Una de las limitaciones encontradas si se piensa en la competencia científica desde un enfoque holístico, corresponde a las diferencias que se aprecian en el desarrollo de cada una de las dimensiones, lo cual revela las dificultades en el progreso integral de la competencia, ya que algunas dimensiones ofrecen mayor dificultad que otras. Por lo cual sería interesante en próximas investigaciones realizar el estudio de una cohorte en el transcurso del ciclo básico.

El impacto, del trabajo con los proyectos de indagación, fue auspicioso, se partió de grupos con rendimientos disímiles y, al final del curso, debido al avance en las distintas dimensiones de la competencia científica, dejaron de apreciarse las diferencias entre grupos del mismo grado. Sumado a ello se debe destacar que las opiniones de docentes y estudiantes sobre la experiencia de trabajar con proyectos fueron en general muy positivas. 